\section{Dyeing to diagnose preeclampsia}

\section{By Michael J. Haas, Senior Writer}

Although there is widespread agreement on the need for a better way to diagnose preeclampsia, few companies have pursued new tests because finding specific markers has been difficult. A Yale-led group has found dye-binding aggregates of misfolded proteins that could be markers for preeclampsia and has developed a simple urine test that can rapidly diagnose the condition. ${ }^{1}$

The test could speed up treatment for women who have preeclampsia and spare unnecessary procedures in women who do not, but it is not yet clear whether the primary market would be in developed or underdeveloped countries.

Early next year, three members of the team behind the study expect to launch an international trial of an inexpensive version of the test that Yale School of Medicine spinout GestVision Inc. will supply.

Preeclampsia is not uncommon-occurring in about $5 \%$ of pregnancies - and can lead to maternal seizures that are life threatening to both mother and fetus. Currently, the
"There is a need for a preeclampsia diagnostic that is not based on subjective assessments of blood pressure and proteinuria but instead is based on objective biochemical markers."

-Irina Buhimschi,

Nationwide Children's Hospital distinguish proteinuria caused by preeclampsia from other causes of high protein in the urine.

In the same study, her team also showed that urine and placentas from women with preeclampsia contained oligomeric aggregates that had misfolded protein fragments of the protease inhibitor $\alpha_{1}$-antitrypsin (AAT; A AT; SERPINA1). ${ }^{3}$

That suggested to Buhimschi and colleagues that preeclampsia could involve multiple oligomers of misfolded proteins-known generically as amyloids. Moreover, their presence in urine could provide an accessible diagnostic marker for the condition.

In the new study, Buhimschi teamed up with researchers from several university hospitals to test whether amyloids could be used to detect preeclampsia. The group identified amyloids from at least six different proteins in urine from patients with preeclampsia that were not found in urine from women with other conditions that cause proteinuria.

Next, the researchers turned to Congo red, a dye frequently used to detect amyloids in vitro and in vivo. They spotted urine samples from pregnant women onto a nitrocellulose strip, treated the strip with Congo red and then washed away unbound dye. The ratio of the color intensity before and after the wash provided a quantitative readout for amyloid levels in the sample.

In a validation cohort of nearly 600 pregnant women, the method diagnosed preeclampsia with $86 \%$ sensitivity and $85 \%$ specificity. In diagnostics, the sensitivity of a test reflects its ability to correctly identify individuals with the disease, whereas the specificity reflects its ability to identify individuals who do not have the disease.

Moreover, the method correctly diagnosed the condition in women who had atypically low levels of proteinuria and readily distinguished only effective treatment is preterm delivery by induced labor or Caesarean section. The standard of care in the U.S. and EU for managing the condition prior to delivery includes antihypertensives to reduce blood pressure, magnesium sulfate to prevent seizures and steroids to promote fetal lung development.

Preeclampsia is usually diagnosed in the second or third trimester when clinical symptoms of hypertension and proteinuria appear. But those measures are of limited help because high blood pressure can be caused by other conditions and preeclampsia can occur without significant proteinuria-referred to as atypical preeclampsia.

As a result, a common outcome of a preeclampsia diagnosis is that the patient is watched closely to see if symptoms worsen, which can delay treatment decisions for several weeks or more.

"There is a need for a preeclampsia diagnostic that is not based on subjective assessments of blood pressure and proteinuria but instead is based on objective biochemical markers," study leader Irina Buhimschi told SciBX.

In 2008, Buhimschi led a study that found proteins in urine from patients with preeclampsia that were not present in urine from women who had uneventful pregnancies or other conditions that cause proteinuria. ${ }^{2}$ The study was the first to identify proteins that could it from other pregnancy-related conditions and comorbidities such as gestational hypertension and proteinuria unrelated to preeclampsia.

Results were published in Science Translational Medicine.

Buhimschi is a professor of pediatrics and obstetrics/gynecology at The Ohio State University College of Medicine and director of the Center for Perinatal Research at Nationwide Children's Hospital.

At the time of the study, Buhimschi was an associate professor of obstetrics/gynecology at the Yale School of Medicine, and her team included researchers from The Ohio State University, the New York University School of Medicine, The University of Vermont School of Medicine, the University of California, Irvine and King Abdulaziz University.

\section{Confirmation bias}

Buhimschi told SciBX that her team developed the technology to address the limits of current diagnostic criteria. The aim was to improve preeclampsia determination by using the test in addition to those criteria or instead of them.

Matthew Cooper, founder and CEO of Carmenta Bioscience Inc., said that the team's approach "is an absolutely feasible way to confirm 


\section{Box 1. Once more into the misfoldome.}

In addition to identifying a method for diagnosing preeclampsia, the report in Science Translational Medicine suggests that the etiology of the disease involves the misfolding of multiple proteins $-\mathrm{a}$ finding the team thinks could eventually lead to a therapy. ${ }^{1}$

The team showed that urine from women with preeclampsia contained amyloids from at least six different proteins that were not found in urine from women with uneventful pregnancies: albumin and $\alpha_{1}$-antitrypsin (AAT; $A_{1}$ AT; SERPINA1), which the team had previously identified; ${ }^{2}$ amyloid precursor protein (APP); ceruloplasmin ferroxidase (CP); interferon- $\alpha$ inducible protein 6 (IFI6); and immunoglobulin-к light chains.

However, "not all of the preeclampsia patients had all of the proteins in their urine," said team leader Irina Buhimschi, who is a professor of pediatrics and obstetrics/gynecology at The Ohio State University College of Medicine and director of the Center for Perinatal Research at Nationwide Children's Hospital.
Moreover, the team tested patient urine with conformation-specific antibodies against each amyloid and found variations in amyloid types and toxicities among the patients with preeclampsia. For example, she said, "we saw that women with milder cases of preeclampsia had aggregates that were less toxic."

The heterogeneity of the urine amyloids might account for differences in clinical presentation among the subtypes of preeclampsia-including mild, severe, early onset, and atypical, Buhimschi said.

She also said that toxic amyloids that affect specific tissues may explain the association of preeclampsia with other gestational conditions such as hemolysis, liver enzymes and low platelets syndrome, which is sometimes considered part of the preeclampsia spectrum, and peripartum cardiomyopathy, for which preeclampsia is a risk factor.

Thus, the team's long-term goal is to translate its findings about amyloid compositions and conformations into a therapy for preeclampsia.

"This could be done in two ways - by inhibiting protein misfolding in general or by inhibiting more specific aggregates and their toxic effects" with conformationspecific antibodies, she said. "The immune activity of the antibodies could also guide vaccine development."

She added, "We think there are more than just the six types of proteins described in our current paper-this was just the tip of the iceberg. We have now compiled a complete catalog of the proteins and fragments found in preeclampsia urine."

Matthew Cooper, founder and CEO of Carmenta Bioscience Inc., said that such a catalog of proteins and their associations with differing subtypes of preeclampsia could help stratify patients. But he cautioned that it is not yet clear whether the misfolded proteins identified by the team were a cause or an effect of the disease.

Nevertheless, "I would love to see therapeutic targets come of this work," he said. "I truly think the preeclampsia research community is on the cusp of understanding the disease process in a way that will yield therapeutic targets." a diagnosis of preeclampsia. Until now we thought we were alone in the wilderness on developing that kind of diagnostic, so it's good to see that other researchers are getting involved." Carmenta has a panel of six undisclosed serum markers in clinical development as a confirmatory diagnostic for preeclampsia.

In addition, Cooper said that it would be worth finding out which dye-binding proteins were involved because Congo red binds native as well as misfolded proteins. "There may be specific misfolded or native proteins in the urine that have exquisite diagnostic properties" that could improve the performance of the test, he said. He added that improving the test's performance "would be critical because they need to show better differentiation between their test and the 8SG Multistix"-a dipstick from Siemens AG that

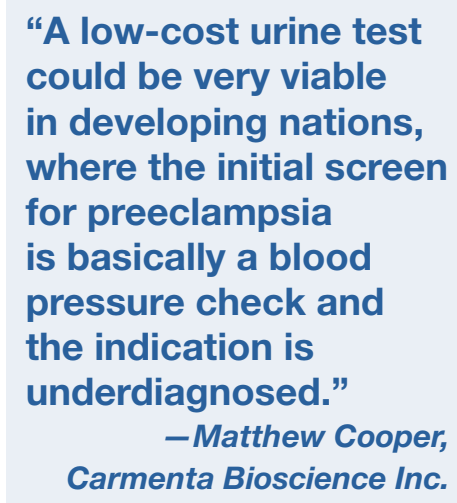

Additionally, she said, the team is still studying the misfolded proteins in urine from preeclampsia patients to identify new therapeutic targets and therapeutic strategies for the disease (see Box 1, "Once more into the misfoldome").

\section{To market, to market}

As Buhimschi and two of her team members prepare for an international clinical trial of the diagnostic, the test's accuracy leaves open the question of whether it is likely to have greater uptake in the developed world or in developing nations.

"The sensitivity and specificity of the test as described in the paper are too low for the developed world, due to the possible repercussions of a false-negative result," Cooper said. But that level of performance "in a low-cost urine test could be very viable in developing nations, where the initial screen for preeclampsia is basically a blood pressure check and the indication is underdiagnosed."

Thus, the two diagnostic approaches-Carmenta's panel of serum markers and the Buhimschi team's urine test-are complementary, he said. "We are working on a test with high performance for use in the developed world first, while Buhimschi and her team are working on a test with lower performance" that could be used primarily in the developing world.

Buhimschi agreed that the clinical setting would determine what level of test performance would be acceptable but disagreed that her team's diagnostic method would be limited to developing nations. "All pregnant women constitute a valid market for a preeclampsia diagnostic 
because there are no gold standards against which the sensitivity and specificity for a preeclampsia diagnostic can be measured," she said.

She added, "We are conducting the trials precisely to find out the sensitivity and specificity that would make a clinically significant difference-and that level of performance might be different than what one would obtain in a laboratory setting."

Wendy Davis, founder and CEO of GestVision, added, "GestVision's initial market for the test would be the U.S., but we are looking at opportunities worldwide" beginning in the three countries-Bangladesh, Mexico and South Africa-where the international trial will take place.

The Yale School of Medicine patented the diagnostic technology developed by Buhimschi's team and spun it out into GestVision. The company plans to commercialize a newer version of the test the team has developed and will begin manufacturing this summer. Buhimschi and Davis declined to disclose the exact form of that version but said that it was suitable for rapid use in point-of-care settings and costs about two cents per test.

In July, Buhimschi's team started a clinical trial of the lab-based version of the test described in the Science Translational Medicine study at an Ohio State University site. Early in 2015 the team will expand the trial to the three international sites using the latest version of the test.

Each site in the trial will enroll about 2,000 pregnant women and will examine whether positive results from the diagnostic resulted in faster treatment for preeclampsia and whether negative results reduced the number of unnecessary preterm deliveries.

Buhimschi said that the trial is not pivotal because it will use a version of the Congo red test that may differ from the final commercial product. However, although it is not designed specifically for regulatory approval, the results could be used to support regulatory applications in the future, Davis said.
Saving Lives at Birth, a consortium of research institutions managed through the U.S. Agency for International Development, is funding the trial.

Buhimschi has no formal affiliation with GestVision, and the company will not be directly involved in the trial.

She said that the team has two papers in review: one describing a cellphone app that makes the colorimetric measurements on the labbased version of the test and the other describing the form of the test that GestVision is commercializing.

Haas, M.J. SciBX 7(30); doi:10.1038/scibx.2014.882

Published online Aug. 7, 2014

\section{REFERENCES}

1. Buhimschi, I.A. et al. Sci. Transl. Med.; published online July 16, 2014; doi:10.1126/scitransImed.3008808

Contact: Irina A. Buhimschi, The Research Institute at Nationwide Children's Hospital, Columbus, Ohio e-mail: irina.buhimschi@nationwidechildrens.org

2. Buhimschi, I.A. et al. Am. J. Obstet. Gynecol. 199, 551.e1-551.e16 (2008)

3. Huntington, J.A. J. Thromb. Haemost. 9, 26-34 (2011)

4. Khurana, R. et al. J. Biol. Chem. 276, 22715-22721 (2001)

\section{COMPANIES AND INSTITUTIONS MENTIONED}

Carmenta Bioscience Inc., Palo Alto, Calif.

GestVision Inc., Guilford, Conn.

King Abdulaziz University, Jeddah, Saudi Arabia

Nationwide Children's Hospital, Columbus, Ohio

New York University School of Medicine, New York, N.Y.

The Ohio State University, Columbus, Ohio

The Ohio State University College of Medicine, Columbus, Ohio

Saving Lives at Birth, Washington, D.C.

Siemens AG (Xetra:SIE; NYSE:SI), Munich, Germany

University of California, Irvine, Calif.

The University of Vermont School of Medicine, Burlington, Vt. U.S. Agency for International Development, Washington, D.C. Yale School of Medicine, New Haven, Conn. 